\title{
Three-mode multiplexer and demultiplexer utilizing trident and multimode couplers
}

\author{
Cao Dung Truong a, Tan Hung Nguyen ${ }^{\text {b,*, }}$ Quang Thai Pham ${ }^{\mathrm{c}}$, Minh Tuan Trinh ${ }^{\text {de,f, }}$, Khu Vu ${ }^{g}$ \\ ${ }^{a}$ Posts and Telecommunications Institute of Technology, Hanoi, Viet Nam \\ ${ }^{\mathrm{b}}$ The University of Danang - University of Science and Technology, Danang, Viet Nam \\ ${ }^{\mathrm{c}}$ Ho Chi Minh City University of Technology, Ho Chi Minh, Viet Nam \\ ${ }^{\mathrm{d}}$ Advanced Functional Materials for Energy and Environmental Applications Research Group, Ton Duc Thang University, Ho Chi Minh City, Vietnam \\ e Faculty of Applied Sciences, Ton Duc Thang University, Ho Chi Minh City, Vietnam \\ ${ }^{\mathrm{f}}$ Department of Electrical Engineering and Computer Science, University of Michigan, USA \\ ${ }^{g}$ Australian National University, Canberra, Australia
}

\section{A R T I C L E I N F O}

\section{Keywords:}

Mode-division multiplexing

Mode conversion

Silicon photonics

Multimode interference coupler

Y-junction coupler

Planar lightwave circuits

\begin{abstract}
A B S T R A C T
In this paper, a simultaneous three-mode silicon (de)multiplexer using a trident coupler and a $3 \times 3$ multimode interference $(\mathrm{MMI})$ is presented. The fundamental $\left(\mathrm{TE}_{0}\right)$, first-order $\left(\mathrm{TE}_{1}\right)$, and second-order $\left(\mathrm{TE}_{2}\right)$ modes at the input are successfully demultiplexed and converted to three uniform fundamental modes $\left(\mathrm{TE}_{0}\right.$ ) at the output ports. The device is designed and optimized by numerical simulation using three-dimensional beam propagation method together with effective index method. The three-mode (de)multiplexing is achieved over the whole Cband with small insertion loss $(<0.9 \mathrm{~dB})$ and crosstalk $(<-17 \mathrm{~dB})$. The proposed device also features with a small footprint $(5 \mu \mathrm{m} \times 400 \mu \mathrm{m})$ and a large fabrication tolerance against chip-size variations while maintaining the desired optical performance.
\end{abstract}

\section{Introduction}

Mode division multiplexing (MDM) has been considered a promising technology to significantly increase the capacity of optical telecommunications [1] as well as photonics on-chip interconnections and computing [2,3]. Since data channels are independently carried on orthogonal eigenmodes of the same wavelength without interchannel crosstalk, overall transmission capacity could be multifold increased in combination with the conventional wavelength division multiplexing (WDM) systems [4,5]. One of the essential elements of MDM systems is mode multiplexer and demultiplexer which are used to convert, combine and divide optical modes at optical transceivers and optical add/drop nodes. To meet the future broadband telecom and on-chip applications, mode (de)multiplexers featuring with small-footprint, wideband, lowloss, small-crosstalk operations and simple fabrication are particularly desirable to realize compact photonic integrated circuits that could work simultaneously for MDM and WDM signals.

In the past few years, different approaches have been used for realizing a mode division (de)multiplexing device. Initial demonstrations used bulky free-space optics [6] which required precise light alignment and caused large power loss and instability. Fiber-based mode (de)multiplexers were also realized by using photonic lantern [7] or cascaded fiber couplers $[8,9]$. However, such fiber-based devices came with large size, large model dispersion due the long propagation, and difficult fabrication. In addition, photonic-crystal mode (de)multiplexer was reported [10], but facing large footprint and challenging fabrication. Another category of mode (de)multiplexers which are based on silicon-on-insulator (SOI) planar lightwave circuits (PLCs) [11,12] was also well investigated. Compared to the previous ones, this class of devices has unique advantages, such as low loss, flexibility, high light confinement, small footprint, and especially, high integration and compatibility with CMOS technology. Different structures of this type including asymmetric directional couplers [13], adiabatic asymmetric couplers [14], cascaded asymmetric Y-junctions [15], and asymmetric Y-splitter [16] were exploited. In principle, such devices could operate with a large number of modes. However, as the number of modes increases, they become difficult for fabrication and longer in footprint caused by the asymmetric and cascaded configurations. Microringbased silicon chip was also reported for mode (de)multiplexing of WDM channels, but the ring structure had narrow operating bandwidth and required delicate fabrication [17]. To overcome the drawbacks of those devices, mode (de)multiplexers utilizing two cascaded multi-mode interference (MMI) couplers [18,19], and a combination of Y-junction and MMI coupler [20] have been proposed, featuring with wideband, low-loss, small footprint and large fabrication tolerance operations. However, these structures supported only two modes and the extension

\footnotetext{
* Corresponding author.

E-mail address: hung.nguyen@dut.udn.vn (T.H. Nguyen).
} 


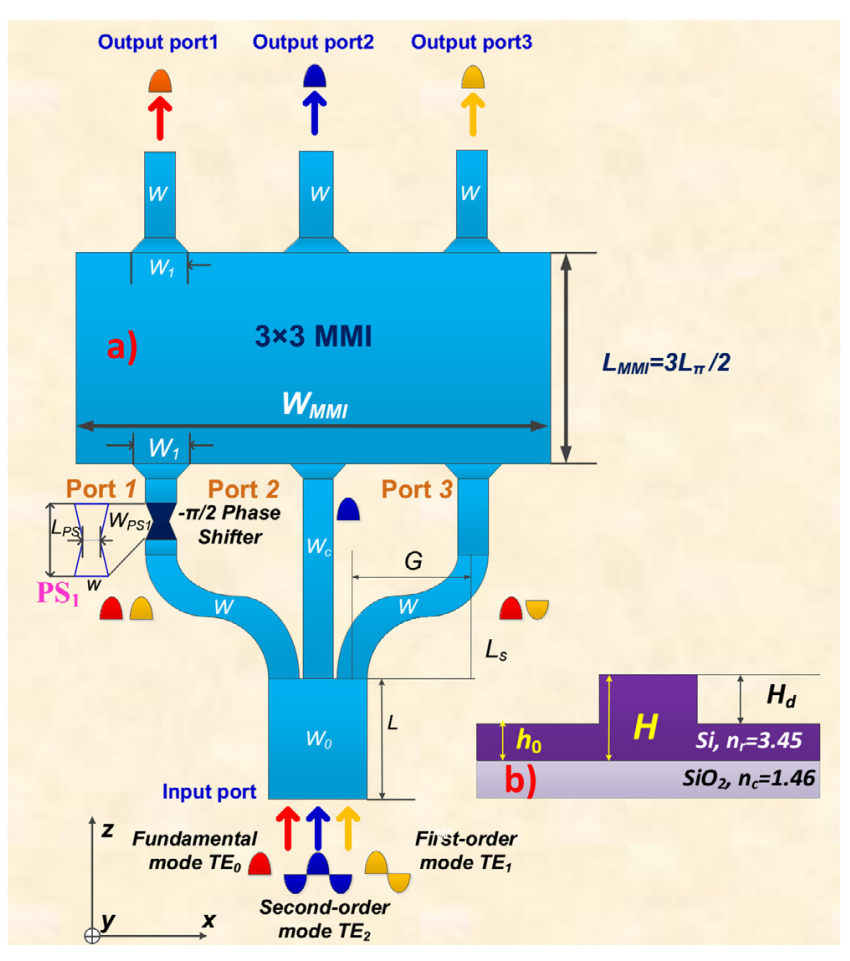

Fig. 1. Schematic of the proposed (de)multiplexer: (a) Top view, (b) Size view.

for using three or more number of modes was impossible due to the nature of the employed configuration. Very recently, a three-mode (de)multiplexer has constructed on a trident junction incorporating with two cascaded MMI couplers [21]. That design used a suitable optical power dividing technique to realize mode (de)multiplexing functions.

In order to reduce the length of the mode (de)multiplexing device, in this paper, we propose and characterize a modified structure of a mode (de)multiplexer supporting three modes from [21] and satisfying all of the aforementioned requirements. The proposed device utilizes a trident $(1 \times 3)$ coupler and a $3 \times 3$ MMI coupler, which are SOI waveguides, to perform simultaneously the mode (de)multiplexing and mode converting of fundamental $\left(\mathrm{TE}_{0}\right)$, first-order $\left(\mathrm{TE}_{1}\right)$, and secondorder $\left(\mathrm{TE}_{2}\right)$ modes to three uniform fundamental modes $\left(\mathrm{TE}_{0}\right)$ at the outputs. Operating mechanism of the proposed device is explained by using transfer matrix relation. Optimization and characterization processes are carried out by using beam propagation method (BPM) together with effective index method (EIM). The three-mode (de)multiplexing is achieved over the whole C-band with small insertion loss $(<0.9 \mathrm{~dB})$ and crosstalk $(<-17 \mathrm{~dB})$. The proposed device also features with a small footprint $(5 \mu \mathrm{m} \times 400 \mu \mathrm{m})$ and a large fabrication tolerance against chipsize variations while maintaining the desired optical performance.

\section{Design and optimization}

\subsection{General description}

Fig. 1 shows the schematic of the proposed (de)multiplexer. It is constituted of a symmetric trident junction coupler with a phase shifter (PS), and a $3 \times 3$ rectangular MMI coupler. Width of the stem waveguide of the trident coupler $\left(W_{0}\right)$ should be wide enough to support the guidance of three modes. This stem is coupled to three spears of narrower waveguides to support only the fundamental mode $\mathrm{TE}_{0}$. While the central waveguide is straight, two outer waveguides are symmetric to each other with respect to the center and have a sinusoidal shape (S-bent waveguide) with the vertical length and the horizontal width of $L_{s}$ and $G$, respectively, as indicated in Fig. 1. One outer arm is connected

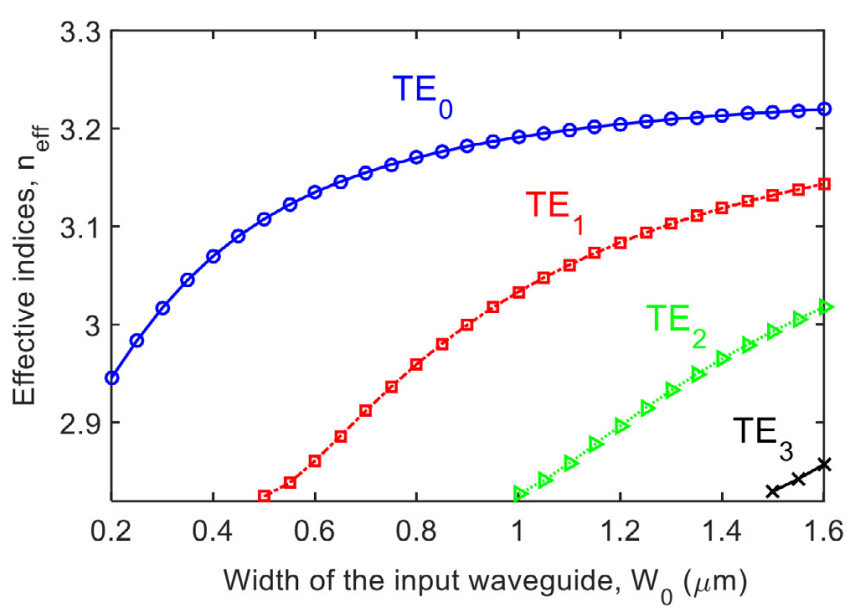

Fig. 2. Effective indices for different modes of the trident coupler as a function of input stem width using BPM method.

with a phase shifter (PS) to make a phase change of $\pm \pi / 2$ so that lights can be properly coupled at the desired outputs of the $3 \times 3 \mathrm{MMI}$. Then, three branches of the trident coupler are connected to the MMI coupler at positions corresponding to center and $\pm W_{M M I} / 3$ of the MMI width. In this design, we set the width of the MMI coupler $W_{M M I}=4.8 \mu \mathrm{m}$.

The device is based on SOI material platform with the forms of sub-micron rib/ridge-type waveguides. The core layer is made of silicon while lower and upper claddings are silica and air, respectively. Refractive indices of the silicon, silica and air layers are selected at $n_{r}=3.45, n_{c}=1.46, n_{\text {air }}=1$ at $1550 \mathrm{~nm}$, respectively. Height of the rib waveguides $H=500 \mathrm{~nm}$ and the slab height $h_{0}=220 \mathrm{~nm}$. Width of the single mode waveguide is selected to meet the single mode condition at wavelength $=1550 \mathrm{~nm}$ [22]. There are some methods to fabricate the proposed device, for instance, the whole device can be fabricated by using the E-beam lithography method and inductively coupled plasma (ICP) etching technique [12]. Optimization of the structure parameters and characterization of the device are implemented through numerical simulation using three dimensional-beam propagation method and effective index method.

\subsection{Trident coupler}

For the trident coupler, we analyze effective indices of the guided modes in silicon rib/ridge waveguides at the input port of the stem. Fig. 2 shows the dependency of effective indices on the width of the trident stem $W_{0}$. It can be seen that when $W_{0}$ varies from $1 \mu \mathrm{m}$ to $1.5 \mu \mathrm{m}$, the trident stem can guide three modes in the waveguide. As a result, we choose $W_{0}=1.4 \mu \mathrm{m}$. The narrow waveguides $(w)$ of the two outer arms of the trident waveguide as well as three output ports of $3 \times 3$ MMI coupler are designed to support only the fundamental mode $\mathrm{TE}_{0}$. Therefore, they are set at $0.5 \mu \mathrm{m}$ for the operation range of single mode regime. This is the upper limit width for cutoff condition of the single mode of TE polarization as seen in Fig. 2. With the above values of $W_{0}$ and $w$, the width of the central arm of the trident coupler $\left(w_{c}\right)$ is determined equal to $0.4 \mu \mathrm{m}$, which also guide only the fundamental mode.

The trident coupler of the proposed device is designed with unique properties with respect to the conventional ones so that it could feature desired functionalities of the three-mode (de)multiplexer. First, because of the naturally symmetric structure of the trident coupler, the firstorder mode $\mathrm{TE}_{1}$ is converted to two fundamental mode $\mathrm{TE}_{0}$ and equally divided to the two outer arms of the trident coupler. Second, the length $L_{s}$ of the sinusoidal waveguides of the trident coupler is adjusted to offer the following functions: (a) when the fundamental mode $\mathrm{TE}_{0}$ is coupled with the stem, the optical field is divided equally into two outer output 


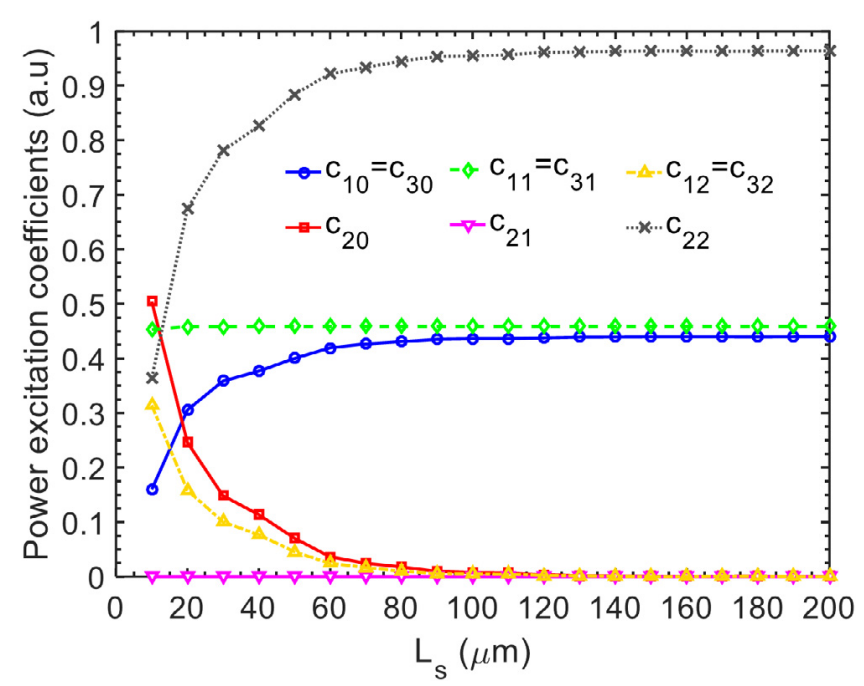

Fig. 3. Power excitation coefficients of the guided mode, when it is led to output ports of the trident coupler, as functions of the length of the S-bent coupler.

waveguides and vanished at the central output waveguide of the trident coupler, and (b) when the second-order mode $\mathrm{TE}_{2}$ is coupled with the stem, the mode is transformed to fundamental mode $\mathrm{TE}_{0}$ and the power is concentrated to the central output waveguide of the trident coupler. Therefore, the power in the central output waveguide for the case of the second order mode equals $\mathrm{TE}_{2}$ is twice that of in the outer arms for the cases of the fundamental mode $\mathrm{TE}_{0}$ and first-order mode $\mathrm{TE}_{1}$. This principle can be addressed thoroughly as follows.

An input field profile $\Psi_{v}(x, 0)$ imposed at $z=0$ at the input of the trident stem ( $x$-coordinate in the direction of the waveguide width while $z$-coordinate in the propagation direction) will be propagated to the end of the stem with the field profile as:

$\Psi_{v}(x, L)=\Psi_{v}(x, 0) \exp \left(j \beta_{v} L\right)$

where $L$ is the stem length of the trident coupler and $\beta_{v}$ is propagation constant at $v$-order mode of the stem $(v=0,1,2)$. This field will be divided into the modal field distribution of all modes $\psi_{m v}(x)$ at output port of the trident coupler as

$\Psi_{\nu}(x, L)=\sum_{m=1}^{3} \varepsilon_{m \nu} \psi_{m \nu}(x)$

where $m$ is the order of output ports of the trident coupler (we denote that $m$ takes $1,2,3$, consecutively from left to right arm). Here, the summation should be understood for both guided and radiated modes. The field excitation coefficients $\varepsilon_{m v}$ of $v$-th mode at $m$ th output port can estimated by using overlap integrals

$\varepsilon_{m \nu}=\frac{\int \Psi_{\nu}(x, L) \psi_{m \nu}(x) d x}{\sqrt{\int \psi_{m \nu}^{2}(x) d x}}$

In the design, we initially set the width of S-bent waveguides of the outer arms of the trident coupler $G=1.15 \mu \mathrm{m}$. This parameter is set accordingly to the device structure described in Section 2.1). By using BPM simulation method, we simulate the power excitation coefficients $c_{m v}=\left|\varepsilon_{m v}\right|^{2}$ at output ports of the trident coupler for each mode $v$. Fig. 3 shows simulated curves of $c_{m v}$ as a function of the length $L_{s}$ of the S-bent waveguide. Simulation results show that when the length $L_{s}$ varies from $10 \mu \mathrm{m}$ to $200 \mu \mathrm{m}$, power excitation coefficients of the fundamental mode $\mathrm{TE}_{0}$ as well as the second-order mode $\mathrm{TE}_{2}$ change rapidly with opposite fashions. As a result, when the optical field of the fundamental mode is evanescent at the central output port, the optical field of the second order mode is concentrated mostly at that port of the trident coupler. In the design, we choose $L_{s}=160 \mu \mathrm{m}$ as an acceptable value so that

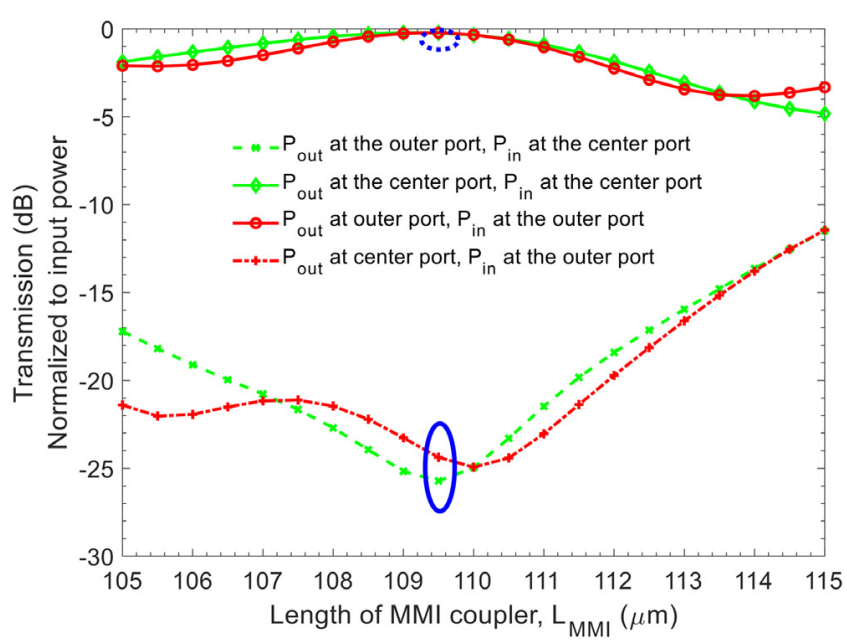

Fig. 4. Transmission characteristic of $3 \times 3 \mathrm{MMI}$ coupler as a function of the length of the coupler.

the trident coupler could achieve an sufficient coupling efficiency for all three modes.

Assume that, the amplitude of the optical fields is denoted in the matrix relation, the transfer matrices for three guided modes of the trident coupler can be presented in turn of the fundamental mode, the first mode and the second mode as follows:

$\mathrm{Y}_{0}=\frac{1}{\sqrt{2}}\left(\begin{array}{c}\mathrm{e}^{\frac{\pi}{2} \mathrm{i}} \\ 0 \\ \mathrm{e}^{\frac{\pi}{2} \mathrm{i}}\end{array}\right) \mathrm{e}^{\mathrm{i} \theta}, \mathrm{Y}_{1}=\frac{1}{\sqrt{2}}\left(\begin{array}{c}\mathrm{e}^{\frac{\pi}{2} \mathrm{i}} \\ 0 \\ \mathrm{e}^{-\frac{\pi}{2} \mathrm{i}}\end{array}\right) \mathrm{e}^{\mathrm{i} \theta}, \mathrm{Y}_{2}=\frac{1}{\sqrt{2}}\left(\begin{array}{l}0 \\ 1 \\ 0\end{array}\right) \mathrm{e}^{\mathrm{i} \theta}$

where $\theta$ is accumulation phase of the optical field of guided modes when propagating through the trident coupler.

\section{3. $3 \times 3$ MMI coupler}

Operating principle of the proposed $3 \times 3 \mathrm{MMI}$ coupler is obeyed Talbot's effect [23]. In the general interference (GI) mechanism [24] of this principle, self-image of a $3 \times 3 \mathrm{MMI}$ coupler will be reformed at the length as $L_{M M I}=3 L_{\pi} / 2$. Here, the half-beat length $L_{\pi}$ of the MMI coupler is given by the relation:

$L_{\pi}=\frac{4 n_{e f f} W_{e}^{2}}{3 \lambda}$

where $W_{e}=W_{M M I}+\frac{\lambda}{\pi}\left(n_{e f f}^{2}-n_{c}^{2}\right)^{-0.5}$ is effective width of the MMI, $\lambda$ is operation wavelength, $n_{\text {eff }}$ is effective index, and $n_{c}$ is refractive index of the cladding. To enhance the transmittance efficiency of the modes, linear tapered waveguides have been placed to link with access waveguides before and after coupling to the MMI region. The length of the MMI region is optimized as shown in Fig. 4 which plots the transmission characteristic of the MMI coupler as a functions of the length $L_{M M I}$. Here, the optimal length is chosen at $L_{M M I}=109.5 \mu \mathrm{m}$ (marked by circles in Fig. 4) for the MMI to achieve a maximum transmission at the desired output ports while keeping a small crosstalk to other ports. This value is agreed with the theoretical length value given in Eq. (5) to achieve maximum transmittance efficiencies for three modes. Similarly, to maximize the light confinement and minimize crosstalk for $\mathrm{TE}_{2}$ mode, the width of linear taper is selected at $0.9 \mu \mathrm{m}$.

Next, we connect three output ports of the trident coupler with the $3 \times 3$ MMI coupler. On one of two outer arms of the trident coupler connected to the MMI coupler, we place a phase shifter (PS) to combine/separate optical paths for the operation process of the (de)multiplexing function. Since the proposed device has a symmetrical structure, without loss of generality, the PS is placed on the left outer arm as seen in Fig. 1. From the symmetric interference mechanism, the 


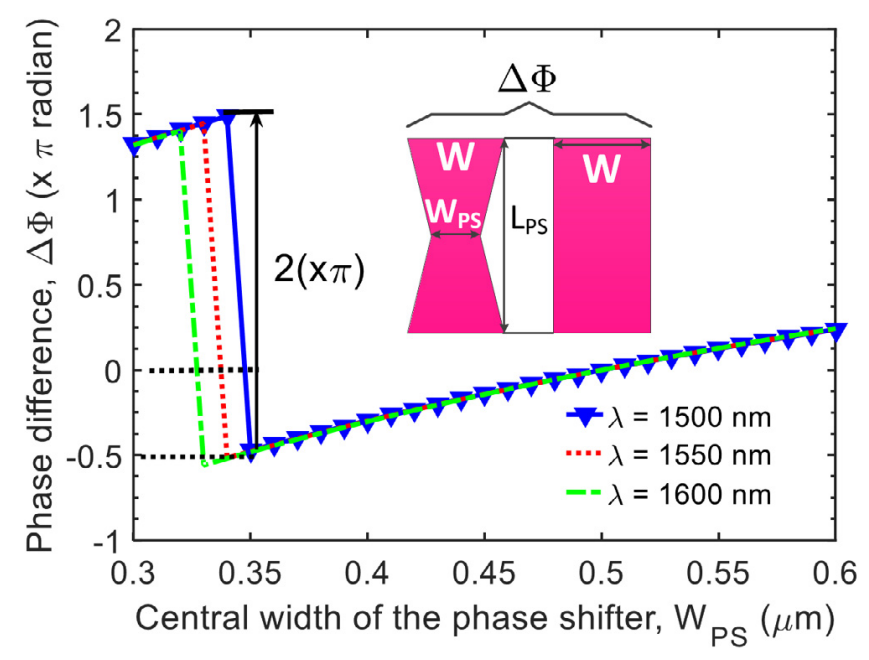

Fig. 5. BPM simulation for the phase shift $\Delta \Phi$ as a function of the central width of the phase shifter $\left(W_{p s}\right)$ for three wavelengths: $1500 \mathrm{~nm}, 1550 \mathrm{~nm}$ and $1600 \mathrm{~nm}$.

self-imaging at the central line of the MMI coupler will be reproduced at the length equally to an integer multiple number of the characteristic length to be $L_{M M I}=3 L_{\pi} / 4$. Thus, the signal at the center input port will be guided continuously to the central output port of the MMI coupler (with the length of $L_{M M I}=3 L_{\pi} / 2$ ).

When the PS produces a phase shift $\Delta \Phi=\pi / 2$, the transfer matrix for the two outer input/output arms of the MMI can be written as $[23,24]$

$\mathrm{Y}_{0}=\frac{1}{\sqrt{2}}\left(\begin{array}{cc}1 & \mathrm{e}^{\frac{\pi}{2} \mathrm{i}} \\ \mathrm{e}^{\frac{\pi}{2} \mathrm{i}} & 1\end{array}\right)$

Therefore, the transfer matrix for $\mathrm{TE}_{0}$ mode when the PS is set at $\pi / 2$ written as

$\mathrm{Q}_{0}=\frac{1}{2}\left(\begin{array}{cc}1 & \mathrm{e}^{\frac{\pi}{2} \mathrm{i}} \\ \mathrm{e}^{\frac{\pi}{2} \mathrm{i}} & 1\end{array}\right)\left(\begin{array}{c}\mathrm{e}^{\pi \mathrm{i}} \\ \mathrm{e}^{\frac{\pi}{2} \mathrm{i}}\end{array}\right) \mathrm{e}^{\mathrm{i} \theta}=\left(\begin{array}{c}-1 \\ 0\end{array}\right) \mathrm{e}^{\mathrm{i} \theta}$

The transfer matrix for $\mathrm{TE}_{1}$ mode when the PS is set at $\pi / 2$ written as

$\mathrm{Q}_{1}=\frac{1}{2}\left(\begin{array}{cc}1 & \mathrm{e}^{\frac{\pi}{2} \mathrm{i}} \\ \mathrm{e}^{\frac{\pi}{2} \mathrm{i}} & 1\end{array}\right)\left(\begin{array}{c}\mathrm{e}^{\pi \mathrm{i}} \\ \mathrm{e}^{-\frac{\pi}{2} \mathrm{i}}\end{array}\right) \mathrm{e}^{\mathrm{i} \theta}=\left(\begin{array}{c}0 \\ -\mathrm{i}\end{array}\right) \mathrm{e}^{\mathrm{i} \theta}$

Similarly, we can also write the transfer matrix for $\mathrm{TE}_{0}$ and $\mathrm{TE}_{1}$ modes when the PS is set at $-\pi / 2$ as follows

$\mathrm{Q}_{0}=\left(\begin{array}{l}0 \\ \mathrm{i}\end{array}\right) \mathrm{e}^{\mathrm{i} \theta}, \mathrm{Q}_{1}=\left(\begin{array}{l}1 \\ 0\end{array}\right) \mathrm{e}^{\mathrm{i} \theta}$

Expressions (7)-(9) prove that the setting of phase shift $\Delta \Phi= \pm \pi / 2$ is an important condition for input signals at $\mathrm{TE}_{0}$ and $\mathrm{TE}_{1}$ modes to be combined or separated into the two individual output ports of the MMI coupler. In this paper, we choose the phase shift $\Delta \Phi=-\pi / 2$ for realizing the function of the mode (de)multiplexing of the device.

\subsection{Phase shifter}

To achieve the phase shift $\Delta \Phi=-\pi / 2$ for (de)multiplexing operation, a butterfly-formed waveguide is used to make a passive phase shifter as shown in the inset of Fig. 5. This PS has the length $L_{p s}=$ $13 \mu \mathrm{m}$ and the width $w=500 \mathrm{~nm}$. The central width $W_{p s}$ of the butterfly waveguide is varied to make the propagation constant changes proportionally. The phase shift over the PS can be calculated by using the following formula:

$\Delta \Phi\left(z_{0}, z_{0}+L_{p s}\right)=\int_{z_{0}}^{z_{0}+L_{p s}}\left[\beta_{0}-\beta_{p s}(z)\right] d z$
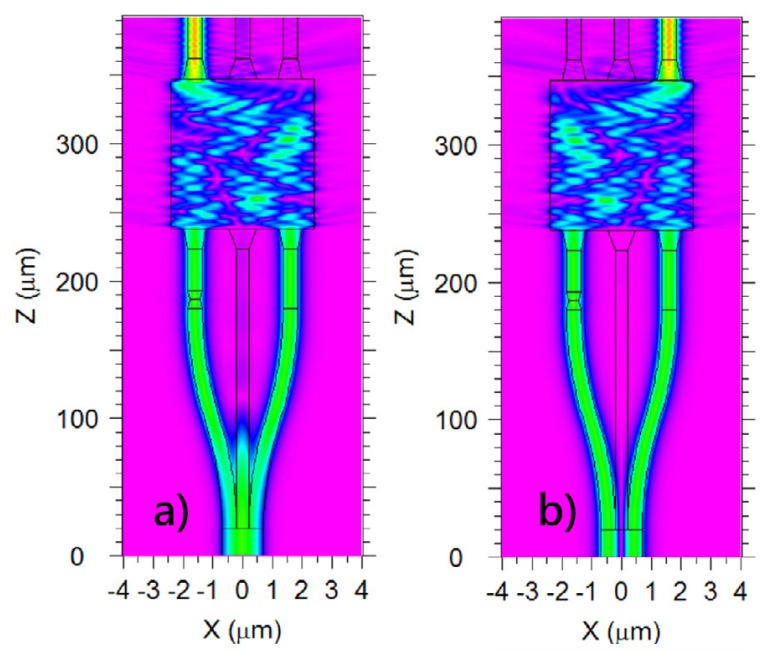

1.0
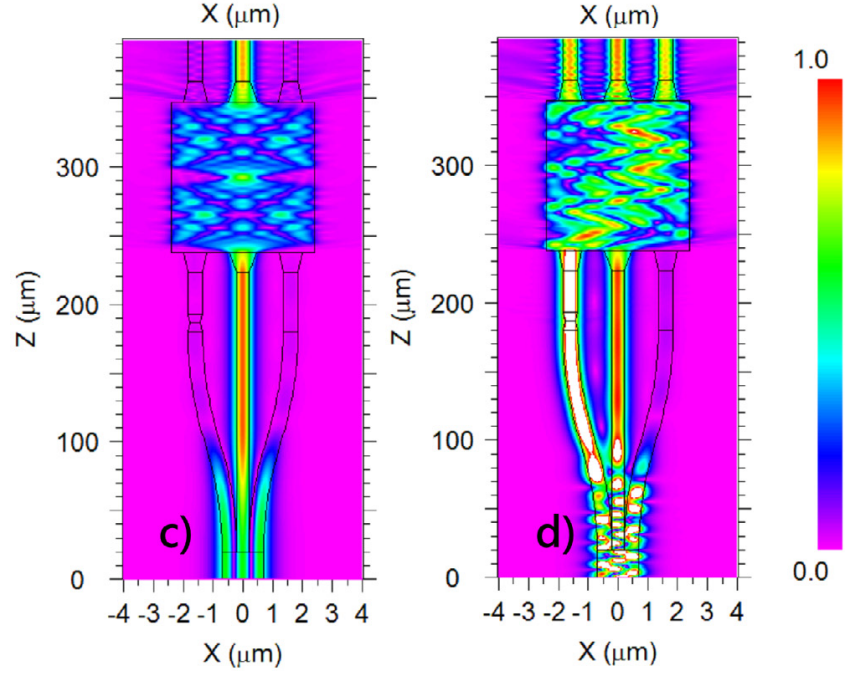

Fig. 6. Electric field patterns of the proposed three-mode (de)multiplexer for fundamental mode (a), first-order mode (b), second-order mode (c), and total of three modes (d). Color bars indicate normalized field intensity. (For interpretation of the references to color in this figure legend, the reader is referred to the web version of this article.)

where $z_{0}$ and $z_{0}+L_{p s}$ are the beginning and ending points of the PS along $z$-direction, respectively; $\beta_{0}$ is the propagation constant at point $z_{0}$ and $\beta_{z}$ is a function of the propagation at $z$-point within $\left(z_{0}, z_{0}+L_{p s}\right)$. Fig. 5 shows BPM simulation for variation of the phase difference $\Delta \Phi$ as a function of the central width $W_{p s}$. We can see that at the width $W_{p s}=0.35 \mu \mathrm{m}$, the phase shift $W_{p s}$ given by the phase shifter is $-\pi / 2$. It is important to note that since the phase shifter is constructed in the form of the tapered waveguide, it is almost insensitive to wavelength [21]. With this design, the fundamental and first-order modes can be demultiplexed to the left and right output arms of the MMI, respectively.

\section{Optical performance and discussion}

3D-BPM numerical simulation together with effective index method is used to examine the optical performance of the designed device. We will observe the optical modes when they are excited and propagated into the device from the input port to the output ports. Fig. 6 shows electric field pattern of the proposed mode (de)multiplexer at wavelength of $1550 \mathrm{~nm}$ when fundamental mode $\mathrm{TE}_{0}(\mathrm{a})$, first-order mode $\mathrm{TE}_{1}(\mathrm{~b})$, second-order mode $\mathrm{TE}_{2}(\mathrm{c})$, and all three modes (d) are launched to the device, respectively. It can be seen that, the optical field of $\mathrm{TE}_{2}$ mode is guided to the central output port while those of $\mathrm{TE}_{0}$ and $\mathrm{TE}_{1}$ modes are divided into the right and left output ports, respectively. Fig. 6 proves a 
successful simultaneous (de)multiplexing of three modes $\mathrm{TE}_{0}, \mathrm{TE}_{1}$ and $\mathrm{TE}_{2}$ as predicted over our simulation and analysis. Small amount of light is radiated out of the waveguide, suggesting a small power loss of the device.

Next, we evaluate the optical performances of the proposed device taking into account important parameters including insertion loss (I.L) and crosstalk (Cr.T) which are defined as

I.L $=-10 \log _{10} \frac{P_{\text {out }}}{P_{\text {in }}}$

Cr.L $=-10 \log _{10} \frac{P_{\text {out }}}{P_{\text {unwanted }}}$

Note that the total loss of the device in practice would be higher due to the power loss of the light coupling between the planar waveguides and optical fibers when the chip is connected to fiber-terminated components of the optical fiber communication system. The light coupling can be realized by using several techniques, for example, using a linear taper waveguide to guide the light between fiber and the chip, or using a grating coupler to convert the momentum from the optical fiber to the planar waveguide. The grating coupler has been widely used for high coupling efficiency between the silicon waveguide and the optical fiber [25].

Fig. 7(a) and (b) show the insertion loss and the crosstalk of the device as a function of wavelength for three modes $\mathrm{TE}_{0}, \mathrm{TE}_{1}$ and $\mathrm{TE}_{2}$. The wavelength is varied within the range from $1500 \mathrm{~nm}$ to $1600 \mathrm{~nm}$. The input power is normalized within the low level to avoid the influence of Kerr effects in silicon waveguides. A small insertion loss and crosstalk are obtained below $0.9 \mathrm{~dB}$ and $-17 \mathrm{~dB}$, respectively, within $40 \mathrm{~nm}$ wavelength range spanning from $1525 \mathrm{~nm}$ to $1565 \mathrm{~nm}$. The mode conversion efficiency changing from $81.3 \%$ to $94.4 \%$ within $40 \mathrm{~nm}$ wavelength range is obtained. The low insertion loss and low modal crosstalk suggest a wide bandwidth operation of the three-mode (de)multiplexer. Note in Fig. 7(a) that the insertion loss of $\mathrm{TE}_{2}$ is always smaller than those of $\mathrm{TE}_{0}$ and $\mathrm{TE}_{1}$ for both short and long wavelength regions due to the designed geometrical parameters of the proposed device. For example, as seen from Fig. 4 , mode $\mathrm{TE}_{2}$, which propagates from the center input port to the center output port of the MMI coupler, has the smallest insertion loss at the center wavelength of $1550 \mathrm{~nm}$. This is because the symmetric interference mechanism results in a smaller loss compared to the asymmetric interference mechanism due to the smaller radiated powers. Furthermore, the access waveguide at the center port of MMI coupler is designed with a narrower width than the outer ports. As a result, the relative ratio of the multimode region width and the access region width for center port is larger than for the outer ones. Consequently, the insertion loss spectrum of $\mathrm{TE}_{2}$ is wider than those of modes $\mathrm{TE}_{0}$ and $\mathrm{TE}_{1}$.

The influence of fabrication errors to the optical performance of the device against geometrical sizes has been also investigated. By scanning geometrical size parameters for each combination of input and output ports, we can quantify the amount of crosstalk which is resulted from the spatial multiplexed and demultiplexed modes. First, Fig. 8(a) show the optical performance of the device against the variation of MMI length $\left(\Delta L_{M M I}\right)$. When $L_{M M I}$ varies within $\pm 0.5 \mu \mathrm{m}$, the insertion loss and crosstalk of the device are smaller than $0.4 \mathrm{~dB}$ and $-18.3 \mathrm{~dB}$, respectively. Second, Fig. 8 (b) show the tolerance of the device to the variation of the input waveguide width $\left(\Delta W_{0}\right)$. As $\Delta W_{0}$ varies in a range within $\pm 50 \mathrm{~nm}$, the insertion loss and crosstalk of the device are smaller than $0.28 \mathrm{~dB}$ and $-19 \mathrm{~dB}$, respectively. For the length of S-bent waveguide which is chosen at $160 \mu \mathrm{m}$, if we varies it gradually around the chosen value, the performance of three modes will change insignificantly as can be see in Fig. 3. Thus, the length tolerance of S-bent waveguide is very large. Moreover, the width of the trident coupler is much wider than that of the access waveguides of MMI coupler, leading to its large tolerance against fabrication error.

Last but not least, the impact of the etching depth variation on the device performance is studied. It should be note that the etching

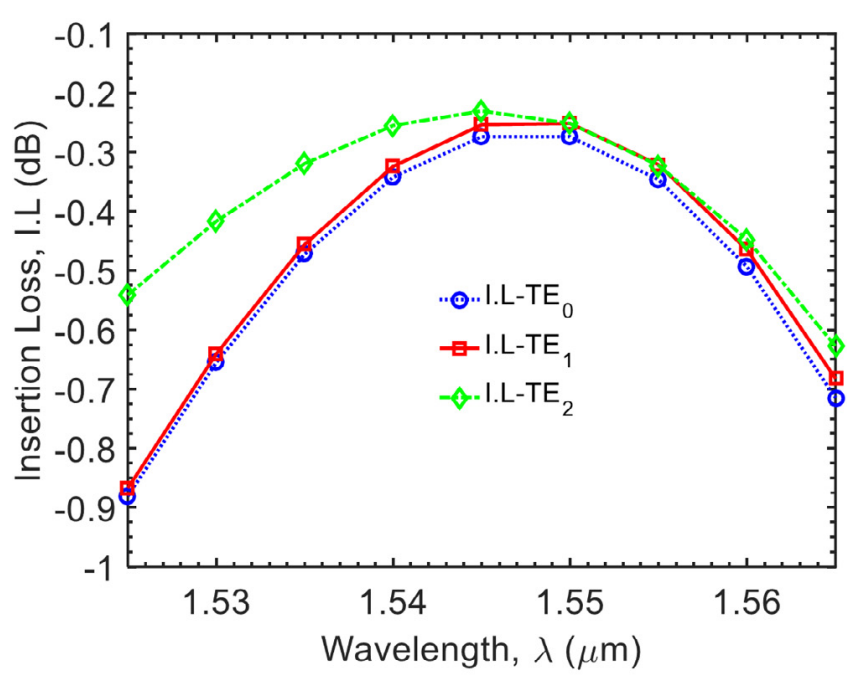

(a)

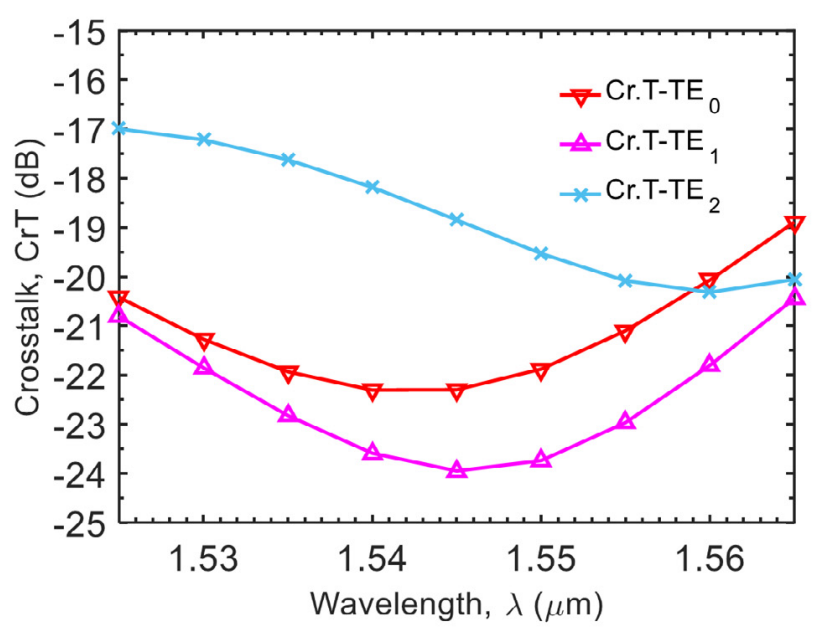

(b)

Fig. 7. Insertion loss (a) and crosstalk (b) of the proposed three mode-(de) multiplexer as a function of the operating wavelength for $\mathrm{TE}_{0}, \mathrm{TE}_{1}$, and $\mathrm{TE}_{2}$ modes.

depth is an important fabrication parameter because it determines the slab thickness of SOI wafer, it is normally difficult to control during fabrication. Fig. 9 plots the insertion loss and crosstalk depending on the etching depth variation. The results show that as the etching depth varies within $\pm 5 \mathrm{~nm}$, the crosstalk is well below the value of $-17 \mathrm{~dB}$ while the insertion loss is below $0.3 \mathrm{~dB}$. These obtained large tolerances of the device are completely suitable to current fabrication technologies, such as E-beam lithography and ICP etching. Compared with the designs in $[18,20,21]$, the present device has comparable optical performance while it successfully works for simultaneous multiplexing of three modes. In addition, the device also exhibits a small footprint as much as $5 \mu \mathrm{m} \times 400 \mu \mathrm{m}$. With the small-footprint, wideband, low loss, low crosstalk and large fabrication tolerance operations, the proposed device could be applied for all-optical (de)multiplexing functions in on-chip silicon photonics integrated circuits.

\section{Conclusion}

We have presented a new structure of a compact three-mode (de)multiplexer using SOI trident-junction and $3 \times 3 \mathrm{MMI}$ couplers. 3DBPM numerical simulation and EIM methods have been put through 


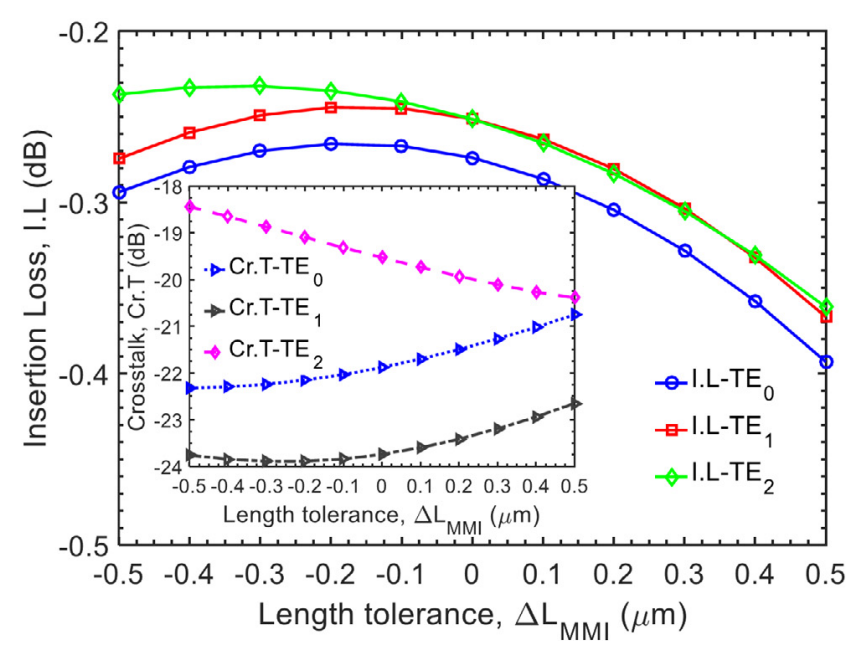

(a)

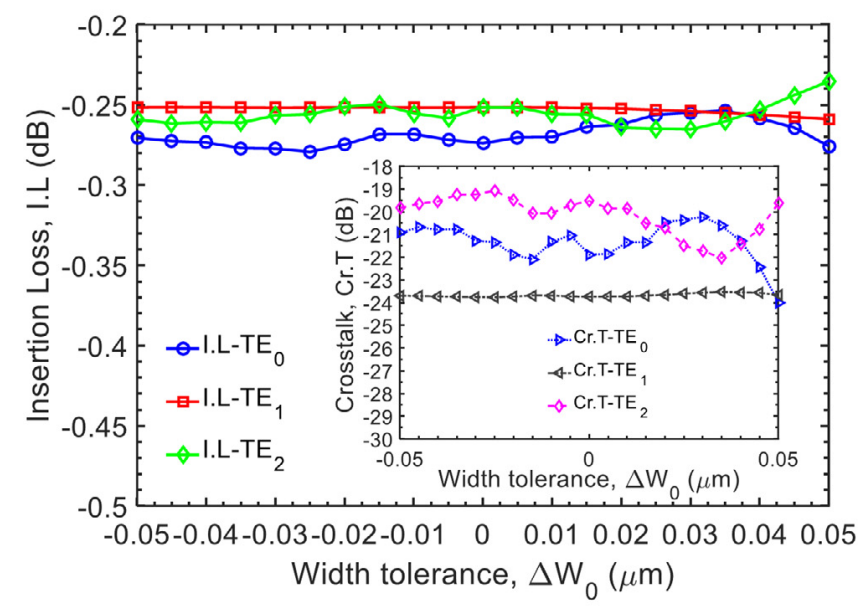

(b)

Fig. 8. Fabrication tolerances of the proposed (de)multiplexer on variation of (a) the second MMI coupler length $\left(L_{M M I}\right)$, and (b) the input waveguide width $\left(W_{0}\right)$

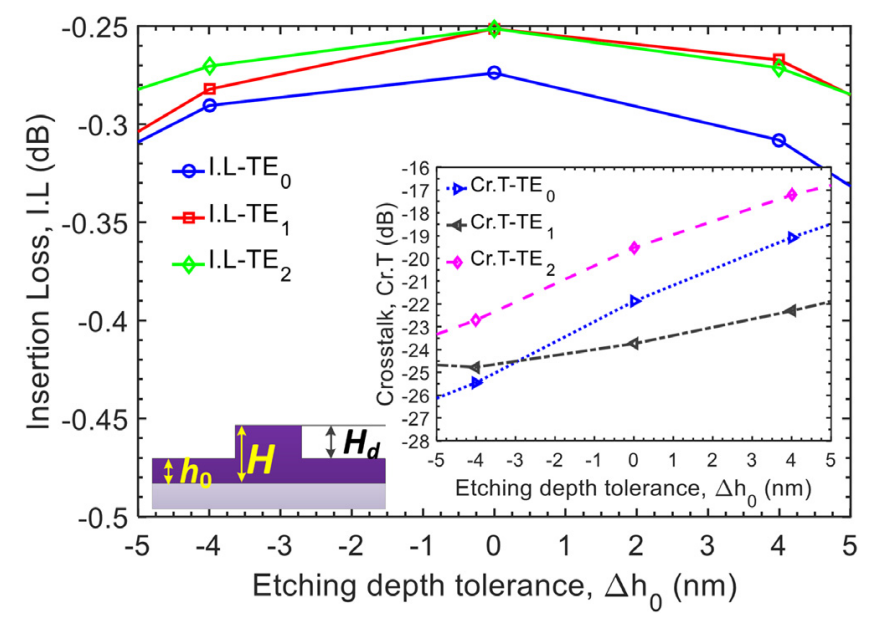

Fig. 9. Insertion loss and crosstalk in the three mode-(de) multiplexer as a function of the etching depth.

to look into the optical performance of the device. All three TE modes $\left(\mathrm{TE}_{0}, \mathrm{TE}_{1}\right.$ and $\mathrm{TE}_{2}$ ) are successfully (de)multiplexed at the outputs with small insertion loss $(<0.9 \mathrm{~dB})$ and crosstalk $(<-17 \mathrm{~dB})$ in the whole
C-band. The device also offers an extra functionality by converting modes of all inputs to an uniform fundamental mode $\left(\mathrm{TE}_{0}\right)$ at the outputs, which made the device compatible with conventional single mode elements at the transceivers. Furthermore, the proposed device feature a small footprint $(5 \mu \mathrm{m} \times 400 \mu \mathrm{m})$ and large fabrication tolerances. With the exemplary features regarding bandwidth, insertion loss, crosstalk, footprint and fabrication tolerance, the present mode (de)multiplexer could be an promising candidate for applications on on-chip photonics integrated circuits and mode multiplexed optical networks.

\section{Acknowledgment}

This research is funded by Vietnam Ministry of Education and Training, Viet Nam under project number B2018.DNA.05.

\section{References}

[1] S. Berdague, P. Facq, Mode division multiplexing in optical fiber, Appl. Opt. 21 (1982) 1950-1955, http://dx.doi.org/10.1364/AO.21.001950.

[2] D.A.B. Miller, Optical interconnects to silicon, IEEE J. Sel. Top. Quantum Electron. 6 (2000) 1312-1317, http://dx.doi.org/10.1109/2944.902184.

[3] R. Nagarajan, et al., Large-scale photonic integrated circuits, IEEE J. Sel. Top. Quantum Electron. 11 (2005) 50-65, http://dx.doi.org/10.1109/JSTQE.2004.841721.

[4] N. Hanzawa, K. Saitoh, T. Sakamoto, T. Matsui, K. Tsujikawa, M. Koshiba, F. Yamamoto, Mode multi/demultiplexing with parallel waveguide for mode division multiplexed transmission, Opt. Express 22 (2014) 29321-29330, http://dx.doi.org/ 10.1364/OE.22.029321.

[5] Z. Zhang, X. Hu, J. Wang, On-chip optical mode exchange using tapered directional coupler, Sci. Rep. 5 (2015) 16072, http://dx.doi.org/10.1038/srep16072.

[6] K. Yoshida, K. Tanaka, T. Tsujimura, Y. Azuma, Assisted focus adjustment for free space optics system coupling single-mode optical fibers, IEEE Trans. Ind. Electron. 60 (2013) 5306-5314, http://dx.doi.org/10.1109/TIE.2012.2218554.

[7] S.G. L.-Saval, N.K. Fontaine, J.R. S.-Gil, B. Ercan, R. Ryf, J. Bland-Hawthorn, Modeselective photonic lanterns for space-division multiplexing, Opt. Express 22 (2014) 1036-1044, http://dx.doi.org/10.1364/OE.22.001036.

[8] N. Bai, et al., Mode-division multiplexed transmission with inline few-mode fiber amplifier, Opt. Express 20 (2012) 2668-2680, http://dx.doi.org/10.1364/OE.20. 002668.

[9] Y. Xie, M.Z.S. Fu, M. Tang, P. Shum, D. Liu, Optimization of few-mode-fiber based mode converter for mode division multiplexing transmission, Opt. Commun. 306 (2013) 185-189, http://dx.doi.org/10.1016/j.optcom.2013.05.016.

[10] A. Sharkawy, S. Shi, D.W. Prather, Multichannel wavelength division multiplexing with photonic crystals, Appl. Opt. 40 (2001) 2247-2252, http://dx.doi.org/10. 1364/AO.40.002247.

[11] A.M.J. Koonen, H. Chen, H. van den Boom, O. Raz, Silicon photonic integrated mode multiplexer and demultiplexer, IEEE Photonics Technol. Lett. 24 (2012) 1961-1964, http://dx.doi.org/10.1109/LPT.2012.2219304.

[12] D. Dai, J. Wang, Y. Shi, Silicon mode (de)multiplexer enabling high capacity photonic networks-on-chip with a single-wavelength-carrier light, Opt. Lett. 38 (2013) 1422-1424, http://dx.doi.org/10.1364/OL.38.001422.

[13] Y.-D. Yang, Y. Li, Y.-Z. Huang, A.W. Poon, Silicon nitride three-mode division multiplexing and wavelength-division multiplexing using asymmetrical directional couplers and microring resonators, Opt. Express 22 (2014) 22172-22183, http: //dx.doi.org/10.1364/OE.22.022172.

[14] S. M.-Garaot, S.-Y. Tseng, J.G. Muga, Compact and high conversion efficiency mode-sorting asymmetric y junction using shortcuts to adiabaticity, Opt. Lett. 39 (2014) 2306-2309, http://dx.doi.org/10.1364/OL.38.001422.

[15] W. Chen, P. Wang, J. Yang, Mode multi/demultiplexer based on cascaded asymmetric y-junctions, Opt. Express 21 (2013) 25113-25119, http://dx.doi.org/10.1364/ OE.21.025113.

[16] J.D. Love, N. Riesen, Single-, few-, and multimode y-junctions, J. Lightwave Technol. 30 (2012) 304-309, http://dx.doi.org/10.1109/JLT.2011.2179976.

[17] L.-W. Luo, et al., WDM-compatible mode-division multiplexing on a silicon chip, Nature Commun. 5 (2014) 1-7, http://dx.doi.org/10.1038/ncomms4069.

[18] T. Uematsu, Y. Ishizaka, Y. Kawaguchi, K. Saitoh, M. Koshiba, Design of a compact two-mode multi/demultiplexer consisting of multimode interference waveguides and a wavelength-insensitive phase shifter for mode-division multiplexing transmission, J. Lightwave Technol. 30 (2012) 2421-2426, http://dx.doi.org/10.1109/ JLT.2012.2199961.

[19] Y. Liu, J. Yan, G. Han, The transmission characteristic of metal-dielectric-metal slot waveguide-based nanodisk cavity with gain medium, IEEE Photonics J. 7 (2015) 1-8, http://dx.doi.org/10.1109/JPHOT.2015.2406766.

[20] Y. Li, C. Li, C. Li, B. Cheng, C. Xue, Compact two-mode (de)multiplexer based on symmetric y-junction and multimode interference waveguides, Opt. Express 22 (2014) 5781-5786, http://dx.doi.org/10.1364/OE.22.005781. 
[21] A.T. Tran, D.C. Truong, H.T. Nguyen, Y.V. Vu, A new simulation design of threemode division (de)multiplexer based on a trident coupler and two cascaded $3 \times 3$ MMI silicon waveguides, Quantum Electron. 7 (2017) 426, http://dx.doi.org/10. 1007/s11082-017-1248-4.

[22] S.T. Lim, C.E. Png, E.A. Ong, Y.L. Ang, Single mode, polarization-independent submicron silicon waveguides based on geometrical adjustments, Opt. Express 15 (2007) 11061-11072, http://dx.doi.org/10.1364/OE.15.011061.

[23] L.B. Soldano, E. C.M.Pennings, Optical multi-mode interference devices based on self-imaging: Principles and applications, J. Lightwave Technol. 13 (1995) 615627, http://dx.doi.org/10.1109/50.372474.
[24] M. Bachmann, P.A. Besse, H. Melchior, General self-imaging properties in $n \times n$ multimode interference couplers including phase relations, Appl. Opt. 33 (1994) 3905-3911, http://dx.doi.org/10.1364/AO.33.003905.

[25] G. Roelkens, D.V. Thourhout, R. Baets, High efficiency grating coupler between silicon-on-insulator waveguides and perfectly vertical optical fibers, Opt. Lett. 32 (2007) 1495-1497, http://dx.doi.org/10.1364/OL.32.001495. 